\title{
EVALI and the Pulmonary Toxicity of Electronic Cigarettes: A Review
}

\author{
Lydia Winnicka, MD and Mangalore Amith Shenoy, MD
}

Department of Pulmonary and Critical Care Medicine, NYU Winthrop Hospital, 222 Station Plaza North, Suite 400, Mineola, NY, USA.

Electronic cigarettes are a novel and emerging product increasingly used by the general public. However, despite their popularity, they remain poorly studied and with likely serious health risks. EVALI, or "electronic cigarette or vaping product use-associated lung injury," is a recently described entity at the forefront of current investigations. Though EVALI has been linked to vitamin $\mathrm{E}$ acetate, a constituent of THC vaping products, electronic cigarettes likely pose a host of other pulmonary toxicities. The presentation, diagnostic work-up, treatment, and pathophysiology of EVALI are herein described, as well as the general pulmonary toxicity profile of electronic cigarettes.

J Gen Intern Med 35(7):2130-5

DOI: $10.1007 / \mathrm{s} 11606-020-05813-2$

(c) Society of General Internal Medicine 2020

\section{INTRODUCTION}

The first electronic cigarette was manufactured in Beijing, China, in 2003 and shortly after, was introduced to the USA in 2006. ${ }^{1}$ Despite still being in its infancy, electronic cigarettes are now a 2.5 billion dollar a year industry. ${ }^{2}$ According to the 2018 National Youth Tobacco Survey, $4.9 \%$ of middle school students and $20.8 \%$ of high school students have used an electronic cigarette in the last 30 days. $^{3}$ Recent prevalence data also shows that current and daily use is steadily increasing, with an alarming $78 \%$ increase in use from 2017 to 2018 among US high school students alone. ${ }^{3,4}$ Though once marketed as a safer alternative to traditional cigarettes, there is growing evidence that these devices are not without risk. There have been 1080 cases EVALI reported to the Center of Disease Control and Prevention (CDC) as of October 1, 2019, including 18 deaths. ${ }^{5}$ The overall pulmonary toxicity profile of electronic cigarettes is now also being better understood. Given the increasing evidence of harm, the use of these products must remain cautioned.

\section{OPERATING PRINCIPLES}

Electronic cigarettes operate on a simple principle of converting a liquid to an aerosol (or "vapor") by the addition

This material has not been previously presented at a national conference.

Received November 14, 2019

Accepted March 19, 2020

Published online April 3, 2020 of heat. ${ }^{6}$ Their use is thus termed "vaping." They are typically made of a metal coil wrapped in a wicking material, which is able to draw in a liquid base. The metal coil is heated by an electric current from a battery, which in turn is able to vaporize the liquid base. ${ }^{6}$ Liquid base is typically composed of a solvent of propylene glycol and vegetable glycerin, with the addition of flavoring, nicotine, and at times, substances such as tetrahydrocannabinol (THC). When heated, both propylene glycol and vegetable glycerin produce a thick, smoke-like vapor. ${ }^{6}$ Though electronic cigarettes bypass the harmful principle of combustion that traditional cigarettes operate on, "vaping" does result in the thermal degradation of liquid base, and dangerous daughter compounds are still produced, including low molecular weight carbonyl compounds (e.g., formaldehyde, acetaldehyde, and acetone) and tobacco-specific nitrosamines. $^{7,8}$

\section{EVALI-DEFINITION AND SYMPTOMS}

Previously published case reports have linked electronic cigarette use to a broad spectrum of pulmonary disease, including asymptomatic and incidental radiographic findings, ${ }^{9}$ lipoid pneumonia, ${ }^{10}$ acute eosinophilic pneumonia, ${ }^{11}$ hypersensitivity pneumonitis, ${ }^{12}$ and diffuse alveolar hemorrhage. ${ }^{13}$ These early case reports were likely the first evidence that electronic cigarettes do carry pulmonary toxicity. More recently, a new entity of respiratory failure due to electronic cigarette use has been described. ${ }^{14}$ This new entity, coined EVALI, initially bewildered clinicians in respect to its cause, though it stressed the temporal relationship between electronic cigarette use and lung disease. A case series describing 53 patients with confirmed or probable EVALI in Wisconsin and Illinois put forth a working definition of respiratory failure with symptom onset within 90 days of electronic cigarette use, with pulmonary infiltrates on imaging, the absence of infection, and no evidence of alternate causes of respiratory failure. ${ }^{14}$

Symptoms of EVALI include shortness of breath, chest pain, cough, and hemoptysis. Gastrointestinal symptoms such as nausea, vomiting, and abdominal pain, and constitutional symptoms such as fever and malaise, are also common. Patients frequently exhibit tachycardia, tachypnea, fever, and hypoxemia at presentation. ${ }^{14}$ The degree of respiratory failure is diverse, with up to one-third requiring intubation and mechanical ventilation. ${ }^{14}$ Demographic analysis of EVALI 
patients found that $67 \%$ are male, the median age is 24 , and up to $86 \%$ are associated with vaping of THC-containing products. ${ }^{15,16}$ A recent survey by the Illinois Department of Public Health, which compared age-matched cohorts of the general public who vaped but did not have EVALI, to patients who did have EVALI, found that EVALI patients had a higher odds of reporting exclusive THC use, frequent THC use ( $>5$ times per day), and obtaining products from "informal" sources, such as off the street, from a dealer, or from a friend. ${ }^{17}$

Pulmonary symptoms can also be found in patients who use electronic cigarettes but do not exhibit EVALI. In one crosssectional study in Hong Kong, symptoms of cough, phlegm, dyspnea, and wheeze were common in electronic cigarette users. ${ }^{18}$ Prior studies have also documented immediate adverse physiologic effects similar to traditional smoking, including increased airflow resistance and a decrease in exhaled nitric oxide. ${ }^{19}$

\section{DIAGNOSTIC WORK-UP}

No laboratory study appears diagnostic, though patients who present with EVALI tend to have leukocytosis with a neutrophilic predominance and elevated inflammatory markers, including ESR, CRP, and procalcitonin. ${ }^{14}$ Peripheral eosinophil count is typically normal. ${ }^{14}$ When bronchoalveolar lavage (BAL) is performed, the cell count often reveals a neutrophilic predominance. ${ }^{14}$ There does appear to be a relationship between electronic cigarettes and acute eosinophilic pneumonia, in which case bronchoalveolar eosinophilia can be seen. ${ }^{11}$ Papanicolaou stain on bronchial washing can show alveolar macrophages laden with vacuoles and oil red $\mathrm{O}$ stain can show lipid-laden macrophages, though the significance of these findings is poorly understood. ${ }^{10,14}$

The CDC, as well as the New York State Department of Health, in conjunction with the University of Rochester Medical Center, have put forth proposed diagnostic algorithms for EVALI. ${ }^{20,21}$ In general, first, a detailed history must be obtained, with attention to respiratory, gastrointestinal, and constitutional symptoms, as well as any recent vaping use in the last 90 days. Information regarding specific brand and flavor of vape, frequency and duration of use, and use of THC should be obtained. Pertinent physical exam findings of fever, tachypnea, and hypoxemia should be noted. Initial laboratory studies should include a complete blood count, a comprehensive metabolic panel, inflammatory markers, and a urine toxicology screen. A chest X-ray should be performed in all patients, with high consideration for a CT chest. Patients should also have concomitant rule-out of infection, with blood cultures, respiratory viral testing including Influenza, HIV testing, and bacterial pneumonia work-up, including Streptococcus and Legionella. Based on clinical discretion, it may also be necessary to rule out mycotic infections, as well as cardiac, rheumatologic, and oncological diseases.
In summary, in a patient with known vaping use in the last 90 days, with a suggestive history and physical exam, classic radiographic findings, and absence of a confounding diagnosis (namely, infection), the diagnosis of EVALI is very likely. Once the diagnosis is established, consultation with pulmonary and/or critical care medicine, infectious disease, and toxicology is recommended, to determine if any additional diagnostic steps should be taken. ${ }^{20,21}$ The use of bronchoscopy with BAL and lung biopsy has no clear established role, but may be warranted on a case-by-case basis (Fig. 1).

\section{RADIOGRAPHIC FINDINGS}

The most commonly seen pattern on CXR and CT chest in Layden et al.'s case series was bilateral, diffuse, ground-glass infiltrates with basilar predominance and sub-pleural sparing. ${ }^{14}$ Diffuse tree-in-bud infiltrates and bilateral nodular infiltrates mimicking metastatic malignancy have also been described. ${ }^{14,22}$ A more recent review of imaging patterns suggests there are actually 4 discrete EVALI radiographic patterns, including acute eosinophilic pneumonia, diffuse alveolar damage, organizing pneumonia, and lipoid pneumonia $^{23}$ (Fig. 2). There is especially substantial overlap between EVALI and exogenous lipoid pneumonia (ELP). ELP is also associated with bilateral ground-glass or consolidative opacities, with lower-lobe predilection. ${ }^{23}$ However, the imaging hallmark of lipoid pneumonia, fat attenuation within opacities in the range of -100 to -3 Hounsfield units, is typically not seen in EVALI. ${ }^{23,}{ }^{24}$ This suggests that inflammation in the lung tissue, which increases the attenuation of the image, plays a large role in EVALI. ${ }^{24}$ Other radiographic presentations of EVALI in the literature include pleural effusions, pneumomediastinum, and pneumothoraces. ${ }^{14,25}$

\section{HISTOPATHOLOGY}

Though the role of biopsy in EVALI remains unclear, in patients that did undergo transbronchial or open lung biopsy in Layden et al.'s case series, findings included nonspecific inflammation, diffuse alveolar damage, infiltration of foamy macrophages, and interstitial and peri-bronchial granulomatous pneumonitis. ${ }^{14} \mathrm{~A}$ more recent review of lung biopsies from 17 patients with EVALI showed patterns of acute lung injury in all cases. ${ }^{26}$ These patterns included acute fibrinous pneumonitis, diffuse alveolar damage, and organizing pneumonia. ${ }^{26}$ In this pathology series, none of the cases reviewed showed histological evidence of ELP. The authors Butt et al. therefore hypothesize that EVALI and ELP are separate entities. ${ }^{26}$ However, as prior case reports have linked electronic cigarette use to ELP and did show cholesterol clefts, it is likely that EVALI and ELP are simply two manifestations of pulmonary toxicity. ${ }^{27}$ As such, the significance of lipid-laden macrophages (either on BAL or on histology) is unclear and may be a marker of exposure and not toxicity. ${ }^{26}$ 


\begin{tabular}{|c|}
\hline Suggested Diagnostic Criteria of EVALI \\
Suggestive symptoms (fever, cough, dyspnea, GI symptoms) \\
\hline Vaping history in past 90 days \\
\hline Suggestive laboratory work (leukocytosis, transaminitis, elevated ESR/CRP) \\
\hline Suggestive imaging \\
\hline Exclusion of infection - everyone \\
\hline Exclusion of cardiac, rheumatologic, and oncologic causes - case by case \\
\hline Bronchoscopy with BAL - case by case \\
\hline Lung biopsy - case by case
\end{tabular}

Figure 1 Suggested diagnostic criteria of EVALI.

\section{PATHOPHYSIOLOGY OF EVALI}

As previously mentioned, the majority of EVALI cases reported are associated with the vaping of THC-containing products. ${ }^{15,16}$ Analysis of samples by the Food and Drug Administration (FDA) identified "vitamin E acetate," used as a thickening agent in THC products, as a likely culprit in the development of EVALI. ${ }^{15}$ The CDC analyzed 29 EVALI-associated BAL samples and found they all contained vitamin E acetate. Together with the previous FDA product testing results, it has been concluded there is a definite association between vitamin E acetate and EVALI. ${ }^{15}$ A subsequent follow-up study also detected vitamin $\mathrm{E}$ acetate in 48 of $51 \mathrm{BAL}$ samples from patients with EVALI, further providing evidence that this is a likely culprit. ${ }^{28}$ When inhaled, vitamin $\mathrm{E}$ acetate is incorporated into the natural phospholipids that comprise surfactant, increasing its permeability and decreasing its functioning. ${ }^{29}$ We postulate that this loss of normal functioning surfactant, which would increase surface tension of alveoli, may also cause an inflammatory cascade in lung tissue. The thermal breakdown of vitamin $\mathrm{E}$ acetate is also poorly understood, and potential harmful daughter compounds may also have a role in EVALI. $^{28}$

The largest number of EVALI hospitalizations occurred during the week of September 15, 2019. ${ }^{16}$ Since then, cases per week have been steadily declining. It is plausible that as more national attention was given to EVALI, law enforcement agencies began confiscating more illicit substances from the streets. This would be in line with the finding that most EVALI patients did obtain their THC-containing products from

\section{Radiographic Features of EVALI}

Diffuse, bilateral, ground-glass infiltrates

Basilar predominance

Sub-pleural sparing

Imaging in-line with AEP, DAD, OP, or ELP

Figure 2 Radiographic feature of EVALI. AEP, acute eosinophilic pneumonia; DAD, diffuse alveolar damage; OP, organizing pneumonia; ELP, exogenous lipoid pneumonia. "informal" sources. Despite the strong evidence that THCcontaining products, and specifically, the constituent "vitamin E acetate," are at the root of EVALI, there remains a small but consistent number of EVALI patients who do not use any THC products. ${ }^{16}$ Additional investigations into the pathophysiology of EVALI are therefore still warranted. ${ }^{16}$

\section{GENERAL PULMONARY TOXICITY}

Though the link between vitamin $\mathrm{E}$ acetate and EVALI is now seemingly clear, there is growing evidence that inhaling electronic cigarette vapor does pose general toxicity (Fig. 3). In vitro studies have demonstrated a dose-dependent decrease in viability of normal human bronchial epithelial cells after exposure to electronic cigarette vapor. There appears to be dose-dependent DNA damage, depletion of glutathione stores, and increase in cell membrane permeability. ${ }^{30}$ Histology studies have shown that when buccal cells are exposed to the liquid base component of electronic cigarettes, there are marked cell alterations, including apoptosis, dyskeratosis, and epithelial atrophy. ${ }^{30}$ Mice that were exposed to electronic cigarette vapor were also found with increased concentrations of cytokines in subsequent BAL samples, including IL-6, MCP-1, IL- $1 \alpha$, and IL-13, signifying inflammatory stress. ${ }^{31}$ Though the implication of these studies is unclear, there appears to be significant potential for the development of lung disease with heavy electronic cigarette use.] $\rightarrow$

The heating coil of electronic cigarettes is also a suspected source of pulmonary toxicity. As the coil undergoes repeated heating and cooling, trace metal molecules can reach the liquid base and subsequently the lung epithelium. ${ }^{6}$ Exposure to fumes containing metal has well-established toxicity, including an association with respiratory tract infections and lung cancer. ${ }^{32}$ Prior studies have noted blood levels of lead, mercury, and copper that are comparable among cigarette-only and electronic cigarette-only users. ${ }^{33,}{ }^{34}$ One additional study found evidence of aluminum, calcium, chromium, copper, iron, lead, magnesium, tin, and zinc molecules in electronic cigarette aerosols. ${ }^{35}$ In addition, the wicks of electronic cigarettes are commonly made of silica, nanoparticles of which have been isolated from vapor plumes. Silica has a wellestablished role in respiratory dysfunction. ${ }^{6}$ 


\begin{tabular}{|ll|}
\multicolumn{1}{c}{ Product constituent } & \multicolumn{1}{c|}{ Mechanism of Lung Injury } \\
& -asthma, cough \\
& -histological damage \\
& -DNA damage \\
& -inflammatory changes \\
& -oxidative damage \\
\hline Vitamin E acetate (contaminant) & -implicated in EVALI \\
\hline Heating coil & -heavy metal toxicity \\
\hline Wicking material & -silica content, silicosis \\
\hline Flavoring & -thermal degradation \\
& -benzaldehyde \\
& -diacetyl \\
\hline Propylene glycol, vegetable glycerin & -thermal degradation \\
& -formaldehyde \\
& -acetaldehyde \\
& -lipid content \\
\hline
\end{tabular}

Figure 3 Mechanisms of lung injury of electronic cigarettes.

Electronic cigarettes also frequently have flavorings added to the liquid base for an enhanced user experience. These flavorings can mimic traditional substances like fruit, chocolate, and mint, with over 8000 flavors now available on the commercial market. ${ }^{6}$ The inhalational toxicity profile of flavorings is poorly understood. In one study, the vapor of cherry-flavored electronic cigarettes was found to contain benzaldehyde, which is also found in cigarette smoke. ${ }^{36}$ Benzaldehyde is a known respiratory irritant and can cause inflammation and edema of the airways. Diacetyl, a chemical constituent of butter flavoring used in microwave popcorn and associated with severe bronchiolitis obliterans known as "popcorn lung," is also commonly found in electronic cigarette flavorings. ${ }^{37}$ Diacetyl is known to downregulate expression of cilia-related genes and decrease the number of overall ciliated cells. ${ }^{38} \mathrm{~A}$ recent study detected diacetyl in 39 of 51 electronic cigarette brands tested, including in such flavors as watermelon, peach, and pomegranate. ${ }^{37}$ Though there have been no reported cases of bronchiolitis obliterans due to electronic cigarette use, given the known toxicity of inhaling diacetyl, the risk likely exists.

Propylene glycol and vegetable glycerin, the solvent of liquid base, do not remain without caution as well. At high temperatures, they both undergo decomposition to low molecular weight carbonyl compounds, including formaldehyde and acetaldehyde, which are common toxicants found in cigarette smoke. ${ }^{6,8}$ Formaldehyde causes mucous membrane irritation, bronchitis, pneumonia, and pulmonary edema. ${ }^{39}$ The lipid content of vegetable glycerin may also have a role in the development of exogenous lipoid pneumonia. ${ }^{10,27}$ Though vegetable glycerin is a sugar alcohol, it is made by heating triglyceride-rich vegetable fat such palm, soy, and coconut oil, and therefore can contain trace amounts of residual triglycerides. The addition of cannabis-derived oils to liquid base is usually the implicated lipid source that leads to the development of lipoid pneumonia; however, sometimes the solvent itself is the only obvious source of lipid. ${ }^{10}$

\section{TREATMENT}

In the case series of patients in Illinois and Wisconsin, most of the patients received systemic glucocorticoids (92\%), with documentation that this leads to improvement in respiratory status in $65 \%$ of cases. ${ }^{14}$ A case series of 5 patients with ELP associated with vaping also improved with a course of glucocorticoids. ${ }^{40}$ This suggests an inflammatory pathway in the development of both EVALI and ELP. Overall, guidelines recommend steroid dose and duration per clinical guidance. ${ }^{20}$,

${ }^{21}$ Currently though, there are no formal treatment guidelines to guide clinicians, and more studies are needed to determine the optimal duration and dosing of steroids.

\section{USE OF ELECTRONIC CIGARETTES IN SMOKING CESSATION}

The use of electronic cigarettes as a cessation tool to stop smoking traditional cigarettes has had considerable attention over the last 10 years. However, prior studies have had mixed results. Studies have shown that electronic cigarettes both can be a successful tool in smoking cessation ${ }^{41,42}$ and have no effect. ${ }^{43}$ Notably, these studies were all done before the entity of EVALI was discovered, as well as in the infancy of research into the toxicity profile of electronic cigarettes. Also concerning is that in prior studies, there are a large number of patients that though did abstain from traditional cigarettes, became habitual electronic cigarette users. ${ }^{41,}{ }^{42}$ We therefore agree with the current $\mathrm{CDC}$ recommendation that all patients should abstain from all electronic cigarette products and that using electronic cigarettes as a cessation tool is not warranted.

\section{CONCLUSION}

At present, there is substantial evidence that electronic cigarettes (with and without THC use) can lead to lung disease, 
including EVALI. There is also substantial evidence that electronic cigarette use may lead to oxidative and inflammatory damage to the lungs. Electronic cigarette vapor is also known to contain heavy metals, dangerous low molecular weight carbonyl compounds, and dangerous flavoring chemicals. Traditional cigarette smoke contains over 7000 chemicals, with over 70 of them known to be carcinogens, but current research suggests that the toxicity profile of electronic cigarettes and traditional cigarettes is actually quite similar. ${ }^{44}$ The caveat is that certainly comprehensive and definitive research is still lacking. The regulatory climate regarding electronic cigarettes is also rapidly changing, largely owning to the increasing research done in this field. This is most recently reflected in the partial ban of flavored electronic cigarettes by the federal government. ${ }^{45}$

Electronic cigarettes are a fast-growing industry, becoming more popular and more accessible to both youth and adults. Though EVALI is seemingly linked to vitamin E acetate in THC-containing vaping products, and the incidence of cases is now declining, the use of electronic cigarettes must continue to be cautioned by clinicians. This certainly remains an emerging field, though due to rising popularity, clinicians must remain at the forefront of this topic.

Corresponding Author: Lydia Winnicka, MD; Department of Pulmonary and Critical Care Medicine NYU Winthrop Hospital, 222 Station Plaza North, Suite 400, Mineola, NY 11501, USA (e-mail: lydiawinnicka@gmail.com).

\section{Compliance with Ethical Standards:}

Conflict of Interest: The authors declare that they do not have a conflict of interest.

\section{REFERENCES}

1. Consumer Advocates for Smoke Free Alternatives Association. A Historical Timeline of Electronic Cigarettes. Accessed 9/9/19. http://www. casaa.org/historical-timeline-of-electronic-cigarettes

2. Surgeon General. Get The Facts. Accessed 9/17/19. https://e-cigarettes. surgeongeneral.gov/getthefacts.html

3. 2018 National Youth Tobacco Survey. A Startling Rise in Youth Ecigarette Use. Accessed 9/17/19. https://www.fda.gov/tobacco-products/youth-and-tobacco/2018-nyts-data-startling-rise-youth-e-cigarette-use

4. Dai H, Leventhal, A. Prevalence of e-cigarette use among adults in the United States, 2014-2018. JAMA. Published online September 16, 2019. doi:https://doi.org/10.1001/jama.2019.15331

5. Centers for Disease Control and Prevention. Outbreak of Lung Injury Associated with E-cigarette Use, or Vaping. Accessed 9/3/19. https:// www.cdc.gov/tobacco/basic_information/e-cigarettes/severe-lung-disease.html

6. Chun LF, Moazed F, Calfee C, et al. Pulmonary toxicity of e-cigarettes. Am J Physiol Lung Cell Mol Physiol 2017; 313(2): L193-L206.

7. Kosmider L, Sobczak A, Fik M, et al. Carbonyl compounds in electronic cigarette vapors: effects of nicotine solvent and battery output voltage. Nicotine Tob Res 2014; 16(10): 1319-1326.

8. Goniewicz ML, Knysak J, Gawron M, et al. Levels of selected carcinogens and toxicants in vapour from electronic cigarettes. Tob Control 2014; 23(2): 133-139.

9. Flower $\mathbf{M}$, Nandakumar $\mathbf{L}$, Singh $\mathbf{M}$, et al. Respiratory bronchiolitisassociated interstitial lung disease secondary to electronic nicotine delivery system use confirmed with open lung biopsy. Respirol Case Rep 2017; 5(3): e00230

10. McCauley M, Markin C, Hosmer D. An Unexpected Consequence of Electronic Cigarette Use. CHEST 2012; 141(4): 1110-1113.

11. Arter Z, Wiggins A, Hudspath $\mathbf{C}$, et al. Acute eosinophilic pneumonia following electronic cigarette use. Respir Med Case Rep 2019; 27: 100825

12. Sommerfeld C, Weiner D, Nowalk A, et al. Hypersensitivity Pneumonitis and Acute Respiratory Distress Syndrome from E-cigarette Use. Pediatrics 2018; 141(6): e20163927

13. Agustin M, Yamamoto M, Cabrera F, et al. Diffuse alveolar hemorrhage induced by vaping. Case Rep Pulmonol 2018; 9724530.

14. Layden JE, Ghinai I, Pray I, et al. Pulmonary illness related to ecigarette use in Illinois and Wisconsin - preliminary report. N Engl J Med 2019; https://doi.org/10.1056/NEJMoa1911614.

15. Blount B, Karwowski MP, Morel-Espinosa M. Evaluation of Bronchoalveolar Lavage Fluid from Patients in an Outbreak of E-cigarette, or Vaping, Product Use-Associated Lung Injury - 10 states, August-October 2019. MMWR Morb Mortal Wkly Rep 2019; 68(45): 1040-1041.

16. Lozier MJ, Wallace B, Anderson $\mathbf{K}$, et al. Update: Demographic, Product, and Substance-Use Characteristics of Hospitalized Patients in a National Outbreak of E-cigarette, or Vaping, Product Use-Associated Lung Injuries - United States, 2019. Morb Mortal Wkly Rep 2019; 68(49): 1142-1148.

17. Navon L, Jones CM, Ghinai I, et al. Risk Factors for E-Cigarette, or Vaping, Product Use-Associated Lung Injury (EVALI) Among Adults Who Use E-Cigarette, or Vaping, Products - Illinois, July-October 2019. MMWR Morb Mortal Wkly Rep 2019; 68(45): 1034-1039.

18. Vardavas CI, Anagnostopoulos N, Kougias M, et al. Short-term pulmonary effects of using an electronic cigarette: impact on respiratory flow resistance, impendence, and exhaled nitric oxide. CHEST 2012; 141(6): 1400-1406.

19. Wang MP, Ho SY, Leung LT, et al. Electronic cigarette use and respiratory symptoms in Chinese adolescents in Hong Kong. JAMA Pediatrics 2016; 170(1): 89-91.

20. Siegel DA, Jatlaous TC, Koumans EH, et al. Update: Interim Guidance for Health Care Providers Evaluating and Caring for Patients with Suspected E-cigarette or vaping, Product Use Associated Lung Injury United States, October 2019. Morb Mortal Wkly Rep 2019; 68(41): 919927.

21. Kalininskiy A, Bach CT, Nacca NE, et al. E-cigarette, or vaping, product use associated lung injury (EVALI): case report and diagnostic approach. The Lancet Respiratory Medicine 2019; 7(12): 1017-1026.

22. Madsen LR, Krarup N, Bergmann T, et al. A Cancer that went up in Smoke. CHEST 2016; 149(3): e65-e67.

23. Henry TS, Kanne JP, Kligerman SJ. Imaging of Vaping-Associated Lung disease. N Engl J Med 2019; 381(15): 1486-1487.

24. Betancourt SL, Martinez-Jimenez S, Rossi SE, et al. Lipoid Pneumonia: Spectrum of Clinical and Radiologic Manifestations. Am J Roentgenol 2010; 194(1): 103-109.

25. Bonilla A, Blair AJ, Alamro SM, et al. Recurrent spontaneous pneumothoraces and vaping in an 18-year-old man: a case report and review of literature. J Med Case Rep 2019; 13(1): 283.

26. Butt YM, Smith ML, Tazelaar HD, et al. Pathology of Vaping-Associated Lung Injury. N Engl J Med 2019; 381(18): 1780-1781.

27. Viswam D, Trotter S, Burge PS, et al. Respiratory failure caused by lipioid pneumonia from vaping e-cigarettes. BMJ Case Rep 2018; pii: bcr2018-224350.

28. Blount B, Karwowski MP, Shields $\mathbf{P}$, et al. Vitamin E Acetate in Bronchoalveolar-Lavage Fluid Associated with EVALI. NEJM 2019 Dec 20. doi: https://doi.org/10.1056/NEJMoa1916433. [Epub ahead of print]

29. Srivastava S, Phadket R, Govil G. Fluidity, permeability, and antioxidant behavior of model membranes incorporated with alpha-tocopherol and vitamin E acetate. Biochemica et Biophysica Acta - Biomembranes 1983; 734(2): 353-362.

30. Iskandar AR, Zanetti F, Marescotti D, et al. Application of a multi-layer systems toxicology framework for in vitro assessment of the biological effects of Classic Tobacco e-liquid and its corresponding aerosol using an e-cigarette device with MESH technology. Arch Toxicol 2019; 93(11): 3229-3247.

31. Chaumont $\mathbf{M}$, van de Borne $\mathbf{P}$, Bernard A, et al. Fourth generation ecigarette vaping induces transient lung inflammation and gas exchange disturbances: results from two randomized clinical trials. Am J Physiol Lung Cell Mol Physiol 2019; 316(5): L705-L719.

32. Antonini JM, Taylor MD, Zimmer AT, et al. Pulmonary responses to welding fumes: role of metal constituents. J Toxicol Environ Health A 2004; 67(3): 233-249. 
33. Jain RB. Concentrations of cadmium, lead, and mercury in blood among US cigarettes, cigars, electronic cigarettes, and dual cigarette-e-cigarette users. Environ Pollut 2019; 251: 970-974.

34. Jain RB. Concentrations of selected metals in blood, serum, and urine among US adult exclusive users of cigarettes, cigars, and electronic cigarettes. Toxicol Environ Chem 2018; 100(1): 134-142.

35. Williams M, Li J, Talbot P. Effects of Model, Method of Collection, and Topography on Chemical Elements and Metals in the Aerosol of TankStyle Electronic Cigarettes. Sci Rep 2019; 9: 13969.

36. Kosmider L, Sobczak A, Prokopowicz A, et al. Cherry-flavoured electronic cigarettes expose users to the inhalational irritant, benzaldehyde. Thorax 2016; 71(4): 376-377.

37. Allen JG, Flanigan SS, LeBlanc M, et al. Flavoring Chemicals in Ecigarettes: Diacetyl, 2,3-pentanedione, and Acetoin in a Sample of 51 Products, including Fruit-, Candy-, and Cocktail-Flavored E-Cigarettes. Environ Health Perspect 2016; 124(6): 733-739.

38. Park HR, O'Sullivan M, Vallarino $\mathbf{J}$, et al. Transcriptomic response of primary human airway epithelial cells to flavoring chemicals in electronic cigarettes. Sci Rep 2019; 9: 1400.

39. Toxnet. Toxicology Data Network. Accessed 9/1/19. https://toxnet.nlm nih.gov/
40. Davidson K, Brancato A, Heetderks P, et al. Outbreak of ElectronicCigarette-Associated Acute Lipoid Pneumonia - North Carolina, JulyAugust 2019. MMWR Morb Mortal Wkly Rep 2019; 68: 784-786.

41. Caponnetto $\mathbf{P}$, Campagna D, Cibella F, et al. EffiCiency and Safety of an eLectronic cigAreTte (ECLAT) as tobacco cigarettes substitute: a prospective 12-month randomized control design study. PLoS One 2013 8(6):e66317-e66317

42. Hajek P, Phillips-Walker A, Przulj D, et al. A Randomized Trial of ECigarettes versus Nicotine-Replacement Therapy 2019; 380: 629-637.

43. Halpern SD, Harhay MO, Saulsgiver $\mathbf{K}$, et al. A pragmatic trial of ecigarettes, incentives, and drugs for smoking cessation. N Engl J Med 2018; 378:2302-2310.

44. Rodgman A, Perfetti TA. The chemical components of tobacco and tobacco smoke. 2nd CRC Press; New York: 2013.

45. Ducharm J. Trump Administration Announces Stripped-Down Regulations on Flavored Vaping Products. Time. January 2, 2020. . https:// time.com/5758004/flavored-vape-ban/

Publisher's Note: Springer Nature remains neutral with regard to jurisdictional claims in published maps and institutional affiliations. 